Die Qualität sicherzustellen ist eine Kernaufgabe der Ärztinnen und Ärzte: Sie wollen ihre Patienten bestmöglich versorgen. Dies war und ist in selbstverständlicher Art und Weise stets ein wesentlicher Bestandteil der ärztlichen Tätigkeit - und wird seit November 2012 durch die von der FMH gegründete Schweizerische Akademie für Qualität in der Medizin SAQM institutionell verankert. Seit Herbst 2016 existiert die Qualitäts-Charta der SAOM, welche das Commitment der Ärzteorganisationen auch fürTransparenz unterstreicht. Erreicht wird dies mittels Bottom-up-Ansatz und Freiwilligkeit. Auch weiterhin auf Bewährtem und Bestehendem aufzubauen, und hier Weiterentwicklungen mittels geeigneter Rahmenbedingungen zu unterstützen, ist der Schlüssel zum Erfolg. Um dies zu erreichen, ist die konstruktive Zusammenarbeit zwischen den Leistungserbringern und Versicherern ein zentraler Erfolgsfaktor.

\title{
Wie kommt Qualität in die Gesundheitsversorgung?
}

\section{Bernhard Güntert}

Prof. Dr. oec. / MHA, Mitglied der Geschäftsleitung des Krankenversichererverbands curafutura und verantwortlich für das Themengebiet Qualität

Bemühungen um Qualitätssicherung in der Gesundheitsversorgung sind uralt. Im Zentrum stand immer der Schutz der Patienten und die Verpflichtung der Health Professionals, wirksame Behandlungsmethoden anzuwenden. Bereits Hammurapi I. (18. Jahrh. v. Chr.) hat in seinem Codex drakonische Strafen für Ärzte vorgesehen, welche Patienten verletzen oder unsaubere Hilfsmittel verwenden [1]. Ob und wie diese qualitätssichernden Strafen gewirkt haben, ist allerdings nicht überliefert. Sicher ist, dass auch heute noch Qualitätsprobleme bestehen. Das Institute of Medicine zeigt in seiner Publikation To Err is Human [2], dass Patienten in überraschend hohem Masse bei Behandlungen geschädigt werden. Folgestudien in verschiedenen Ländern bestätigten leider diesen Befund (u.a. [3]). Auch in der Schweiz errechnete man, dass - je nach Annahmen - jährlich zwischen 700 und 3000 vermeidbare Todesfälle durch Spitalbehandlungen verursacht werden [4].

\section{Medizin als Wissenschaft und Profession}

Medizin ist eine eigenständige Wissenschaft und Profession. Merkmal von Professionen sind, dass sie Tätigkeitsbereich und erbrachte Leistungen, Aus- und Weiterbildung sowie Zulassung zur Berufsausbildung selbst definieren und zudem über die eigene Tätigkeit forschen [5]. Diese Merkmale trafen auch auf die Medizin zu. Die aktuelle Gesetzgebung zum Gesundheitsund Bildungswesen relativiert diese Selbstbestimmung jedoch.

Zur professionellen Selbstregulierung gehört(e) auch die Definition der Qualität. Eine wichtige Grundlage dazu bildete das Mitte der 60er Jahre entwickelte Qualitätsmodell von Avedis Donabedian. In einem zweidimensionalen Raster unterschied er einerseits Struktur-, Prozess- und Ergebnisqualität und anderer- seits - etwas weniger bekannt, jedoch immer wichtiger werdend - die technische, die interpersonelle und die moralische bzw. ethische Qualität [6]. Dieses Modell dominiert aufgrund seiner Plausibilität die Diskussion um Qualität im Gesundheitswesen noch heute, obwohl die postulierte Kausalität zwischen Struktur, Prozess und Ergebnis empirisch nicht bzw. nur bei sehr grossen Strukturunterschieden nachgewiesen werden konnte.

Einen weiteren Rahmen spannt die Definition des Institute of Medicine: «Quality of care is the degree to which health services for individuals and populations increase the likelihood of desired health outcomes and are consistent with current professional knowledge» [7]. Damit wird der Bezug einerseits zu Evidenz und damit zur immer wichtiger werdenden Diagnose- und Indikationsqualität sowie andererseits zur Bevölkerung geschaffen. Mit dem Bevölkerungsbezug wird die Definitionsmacht der ärztlichen Profession, was unter guter Leistungsqualität zu verstehen ist, relativiert. Qualitätsaspekte der Bevölkerung bzw. der Patienten sowie der Politik und der Versicherer werden mit einbezogen. Politik und Versicherer beeinflussen über die Finanzierung des Gesundheitssystems die verfügbaren Ressourcen und den Zugang zu Leistungen entscheidend. Auch in der Schweiz wurde mit dem Krankenversicherungsgesetz (KVG) von 1996 die professionelle Selbstregulierung eingeschränkt. Mit KVG Art. 58 bekam der Bundesrat die Möglichkeit, Qualitätssicherung zu überprüfen und aktiv anzugehen. Allerdings begnügte er sich damals, die Vertragspartner zu beauftragen, die Qualitätsthemen vertraglich miteinander zu regeln. Das Potential dieser Kompetenzdelegation wurde von den Tarifpartnern jedoch aus verschiedenen Gründen nicht wirklich genutzt. Dies führte u.a. dazu, dass Anhang 6 des TARMED, der die spezifischen Qualitätserfordernisse regeln sollte, nie ausgearbeitet 
wurde. Aber auch andere Bereiche der Selbstregulierung der Health Professionals wurden relativiert. So regelt heute der Staat die Ausbildung und Zulassung der Health Professionals weitgehend allein (KVG Art. 35-40).

\section{Vielfältige Bemühungen um Qualitäts- sicherung}

10 Jahre nach In-Kraft-Setzung des KVG liess das Parlament die Umsetzung der Qualitätssicherung im schweizerischen Gesundheitswesen überprüfen [8]. Die Bemühungen der Vertragspartner wurden als ungenügend bewertet. In der Folge arbeitete der Bund eine Qualitätsstrategie aus [9], in welcher er die Führungsrolle in diesem Bereich beschrieb. Er beabsichtigte, die notwendigen Voraussetzungen für Qualitätssicherung und -entwicklung zu schaffen, Aktionsprogramme zu definieren und die Umsetzung zu sichern. 2014 wurde vom Bund vorgeschlagen, die Aktivitäten der Qualitätssicherung und -entwicklung sowie die der Technologiebewertung in einem «Zentrum für Qualität in der obligatorischen Krankenversicherung» zu konzentrieren [10]. Dieser Ansatz wurde von den meisten Akteuren im Gesundheitswesen abgelehnt. In der Folge übertrug der Bund dem BAG die Verantwortung für HTA. Damit übernimmt der Bund eine aktive Rolle zur Sicherung der Qualität des Leistungskataloges [11]. Ende 2015 unterbreitete der Bundesrat dem Parlament einen neuen Entwurf zur Revision von KVG Art. 58 und 59. Kernstücke dieser Revision waren die Schaffung einer Eidgenössischen Kommission für Qualität in der Krankenversicherung und die Schaffung einer Organisationseinheit am BAG für die Umsetzung von Aktivitäten. Die Finanzierung der Qualitätsstrategie des Bundes sollte durch einen Prämienaufschlag auf die obligatorische Krankenversicherung finanziert werden. Die vorberatende ständerätliche Kommission ist kürzlich nicht auf diesen Vorschlag eingetreten. Und die vorberatende Kommission des Nationalrates hat die Ausarbeitung mehrerer Varianten (aussserparlamentarische Kommission, ad hoc unabhängige Einrichtung) verlangt. Derzeit laufen diese Beratungen noch.

Die aktuelle Diskussion um die Revision von KVG Art. 58 und 59 unter dem Titel «Stärkung von Qualität und Wirtschaftlichkeit» wird kontrovers geführt. Sie zeigt die unterschiedlichen Auffassungen zwischen Health Professionals, Bund, Kantonen und Versicherern bezüglich Qualitätssicherung und -entwicklung im Gesundheitswesen. Grundsätzlich geht es um folgende Fragen: Kann die Leistungsqualität - ähnlich wie in Branchen mit vorwiegend technischer Qualität - top down durch gesetzgeberische Massnahmen verordnet werden? Oder handelt es sich bei Gesundheitsleistungen aufgrund der bedeutsamen interpersonellen und moralischen Aspekte doch um spezielle Leistungen und Situationen? Muss sich Qualität nicht bottom up, im professionellen bzw. heute im interprofessionellen Diskurs und unter Beteiligung der Patienten und der Versicherer entwickeln? Soll der Staat seine Bemühungen, die Leistungsqualität sicherzustellen, nicht darauf fokussieren, Strukturen zu schaffen oder Anreize zu setzen, die eine Auseinandersetzung der Tarifpartner und der verschiedenen Akteure mit Qualitätsfragen im Gesundheitswesen erleichtern, fördern oder auch notwendig machen?

\section{Die Rolle der Health Professionals}

Erfahrungen aus dem In- und Ausland zeigen, dass Qualitätsentwicklungen im Gesundheitswesen i.d.R. durch Health Professionals erfolgen, dass diese jedoch mittels geeigneter Rahmenbedingungen und Verpflichtungen der Akteure gestärkt werden können. So hat etwa die Einführung und Verbreitung des DRGSystems (diagnosebezogene Fallpauschalen) zur Finanzierung der Spitäler in verschiedenen Ländern dazu geführt, dass sich Health Professionals selbst vermehrt Gedanken zu den Prozessen und Behandlungsstandards gemacht haben [12]. Folge davon war, dass Prozess- und Ergebnisqualitätsindikatoren entwickelt und gemessen wurden. Aufgrund der Erfahrungen in den USA und in einigen anderen Ländern wurden in Deutschland vor der Einführung des G-DRG-Systems (2005) die Spitäler bereits 1998 zu Qualitätsmanagement verpflichtet [13]. Die Wahl des QM-Modells (KTQ, proCumCert, IJCQ, EFQM, ISO u.a.) wurde den Häusern überlassen. Seit 2005 sind die Krankenhäuser in Deutschland jedoch verpflichtet, Qualitätsindikatoren und -informationen in Form von Qualitätsberichten regelmässig im Internet zu veröffentlichen. Die vom Gemeinsamen Bundesausschuss vorgegebenen Standards für die Qualitätsberichte werden laufend erweitert. Sie haben die Transparenz im Bereich der stationären Versorgung gefördert und zur Verbreitung von Krankenhaus-Suchmaschinen geführt. Auch in der Schweiz hat der Bund vor Einführung des DRG-Systems (2012) eine Qualitätsstrategie formuliert [9]. In der Folge wurden in einem ersten Schritt zwei nationale Pilotprojekte "Sichere Chirurgie» und «Medikationssicherheit bei Schnittstellen» lanciert. Die bereits 2003 gegründete und breit abgestützte Stiftung für Patientensicherheit (www.patientensicherheit.ch) wurde mit der Umsetzung beauftragt. Eine weitere Initiative im stationären Bereich war die Gründung des Vereins ANQ (www.anq.ch) durch die Kantone, $\mathrm{H}+$ und die 
Versicherer sowie der Abschluss des Nationalen Qualitätsvertrages, welcher die Messung und Publikation verschiedener Qualitätsindikatoren im stationären Sektor ermöglichte. Damit wurden auch in der Schweiz erste Grundlagen für einen Qualitätswettbewerb im Spitalbereich geschaffen. Dass gute Qualität auch Einfluss auf die Base Rate im DRG-System haben soll, wurde für den Moment allerdings durch einen Gerichtsentscheid noch unterbunden [14].

\section{Ambulante Versorgung}

Im Bereich der ambulanten Versorgung verliefen die Anstrengungen um Qualitätsentwicklung und -sicherung in den meisten Ländern weniger zielgerichtet. Die grosse Kritik an den nach der KVG-Revision von 1996 entstandenen Managed-Care-Organisationen in der Schweiz hat dazu geführt, dass sich diese intensiv mit Qualitätsfragen und speziellen QM-Modellen für Arztpraxen und Ärztenetze auseinandergesetzt haben. 1999 wurde die Stiftung EQUAM für Qualitätsarbeit in Hausarztpraxen mit einem spezifischen Fokus auf Managed Care gegründet. EQUAM (www.equam.ch) entwickelt Qualitätsprogramme für Praxen, Ärztenetze und krankheitsorientierte Module für Ärztinnen und Ärzte und passt diese laufend neuen Erfordernissen (z.B. Integrierte Versorgung) sowie an das Europäische Praxisassessment (EPA) an (www.epa-qm.de). Die ursprünglich von Ärzten ausgehende Initiative EQUAM schliesst heute Patientenorganisationen und Versicherer mit ein, ist breit anerkannt (Bund, SAMW usw.), entspricht den Zertifizierungskriterien der Schweizerischen Akademie der medizinischen Wissenschaften und ist eine vom Bund akkreditierte Zertifizierungsstelle [15]. Neben EQUAM entstanden aus der Ärzteschaft heraus verschiedene weitere QM-Programme für die ambulante Versorgung, mit zum Teil sehr unterschiedlichem Fokus, so u.a. QBM (www.politikpatient.ch), Good Medical Practice (GMP) (www.sqs.ch/de/Leistungsangebot/ Produkte/Labels/Good-Medical-Practice), MehrFachArzt MFA (www.mehrfacharzt.ch). Alle diese Q-Modelle und Labels tragen zum Qualitätsbewusstsein bei, achten auf Erfüllung von Qualitätsvorgaben und messen bestimmte Qualitätsindikatoren. Primäre Ziele sind i.d.R. Sensibilisierung der beteiligten Ärzte und Mitarbeiter, Klärung der Basisstrukturen von Arztpraxen und ein internes Benchmarking, um die Qualitätsverbesserungsprozesse bei den teilnehmenden Praxen zu initiieren. Diese Initiativen verfolgen weniger das Ziel, Qualitätstransparenz für Dritte zu schaffen, und können deshalb in dieser Form nicht Basis für einen Qualitätswettbewerb im ambulanten Bereich sein. Auch sind die Konzepte untereinander wenig vergleichbar. Mit der
Q-Initiative gründeten 2013 mehrere dieser Labels eine Plattform mit dem Ziel eines gemeinsamen Auftritts und einer möglichen Harmonisierung. Diese Initiative wurde jedoch nicht weiterverfolgt. Das Anliegen der Harmonisierung wurde von der SAQM aufgegriffen und 2016 mit der Qualitäts-Charta - die zu Transparenz, Nachhaltigkeit und Verbindlichkeit verpflichtet und inzwischen von 67 Ärzteorganisationen unterzeichnet wurde - auch umgesetzt (http://www.fmh.ch/saqm/ qualitaets_charta.html).

Obwohl die Stiftung für Patientensicherheit einen deutlichen Fokus auf die Spitalversorgung legt, hat sie in den vergangenen Jahren auch im Bereich der ambulanten Versorgung verschiedene Qualitätsprobleme identifiziert, insbesondere in Zusammenhang mit Medikationssicherheit, Risikomanagement, Schnittstellenproblemen und Umgang mit Fehlern. Dazu wurden auch Lösungsansätze und Umsetzungskonzepte erarbeitet. Es zeichnet sich ab, dass im Zusammenhang mit Qualitätstransparenz vermehrt weitere Anbieter auf den Markt kommen werden und bspw. Plattformen zur Bewertung von Ärzten oder Arztpraxen anbieten (vgl. u.a.: http://docapp.ch) oder durch den innovativen Einsatz der Informationstechnologie neue Qualitätsinformationen generieren. Stellvertretend sei hier das Projekt "Patient Centered Outcome Registry» (PCOR) von SAQM/FMH mit DiePost (http://www.fmh.ch/ saqm/qualitaetsprojekte.html) genannt. Dieser Ansatz unterscheidet sich von den meisten anderen QM-Konzepten darin, dass im Zentrum das Ausmass der Erreichung der zwischen Patient und Health Professionals festgelegten Behandlungsziele steht und diese mit klinischen Daten in Beziehung gesetzt werden.

Die Bedeutung der oben beschriebenen Labels gegenüber Dritten (Patienten, Versicherern) hat in den letzten Jahren eher etwas abgenommen. Die Krankenversicherer definieren zunehmend über die mit Leistungserbringern abgeschlossenen Managed-Care- und Integrated-Care-Verträgen eigene Anforderungen an Struktur- und Prozessqualität, zunehmend auch an Ergebnisqualität, und vereinbaren die Qualitätsdokumentation. In diesem Rahmen wird somit der gesetzliche Auftrag durch die Tarifpartner zunehmend doch wahrgenommen.

Mit der aktuellen Revision von KVG Art. 58 soll die Qualitätsentwicklung und -transparenz gefördert werden. Leistungsqualität darf dabei nicht mehr nur Sache der Health Professionals sein. Patienten verlassen sich bei der Arztwahl immer weniger alleine auf Mund-zuMund-Empfehlungen oder Zufall, sondern möchten wie in vielen anderen Lebensbereichen auch - objektivierte Informationen über die Leistungserbringer und die Qualität ihrer Arbeit in ihre Entscheidungen 
mit einfliessen lassen. Auch Krankenversicherungsprodukte beinhalten immer häufiger eine Beratungsleistung. D.h. Krankenversicherer benötigen ebenfalls objektivierte Qualitätsinformationen. Während derartige Informationen für Spitäler immer besser verfügbar sind, fehlen sie im ambulanten Bereich noch weitgehend. Sowohl «Die Neuen Patienten» $[16,17]$ als auch die Versicherer werden dies immer mehr einfordern. Die Health Professionals sind daher gefordert, einerseits die bestehenden Modelle transparent zu machen und bei der Entwicklung geeigneter Qualitätsindikatoren, welche auch der eigenen kontinuierlichen Qualitätsentwicklung dienen, mitzuwirken und andererseits die Nutzer in der Interpretation zu unterstützen [18]. Ansonsten wird es sehr wahrscheinlich sein, dass Indikatoren, deren Messung und Publikation top down verordnet werden.

\section{Bottom-up-Qualitätsentwicklung und Transparenz fördern}

Dass der Bund die heutige Situation ändern und den Qualitätswettbewerb im Gesundheitswesen fördern möchte, zeigt die vorgesehene Gesetzesrevision deutlich. Allerdings gilt es einige Punkte zu beachten: Es besteht die Gefahr, dass zentralistische Lösungsansätze, verordnete Qualitätsaktivitäten und -messungen und die Vorgabe nationaler Qualitätsverträge die bestehenden Qualitätsinitiativen mehr behindern als fördern. So hat sich in Deutschland gezeigt, dass die vom Gemeinsamen Bundesausschuss verordneten und von den Kassenärztlichen Vereinigungen umgesetzten Qualitätsaktivitäten und -messungen zu keiner nachweisbaren Verbesserung geführt haben [19]. Einerseits wird die Sinnhaftigkeit von vielen Health Professionals angezweifelt, andererseits ist die Evaluationsbasis angesichts der hohen Komplexität, der vielen Kontextvariablen und der Heterogenität von Qualitätsinterventionen für allgemeine Aussagen schwach. Aus diesen Gründen kommen regionalen oder leistungs- und patientengruppenbezogenen Q-Aktivitäten weiterhin eine grosse Bedeutung in der Qualitätsentwicklung im Gesundheitswesen zu. Stellvertretend für viele andere sei hier auf die "Qualitätsstrategie der stationären Versorgung im Kanton Zürich 2017-2022» hingewiesen (http://www.vzk.ch/fileadmin/PDF/Allgemein/ QualitaetsManagement/Qualitätsstrategie_Kanton_ Zürich.pdf). Derartige Initiativen bringen die Q-Diskussionen zwischen den verschiedenen Akteuren weiter, führen zu neuen Qualitätsindikatoren und Modellversuchen und können, wenn sie sich bewähren, Basis für
Nationale Qualitätsverträge sein. Es ist daher zu wünschen, dass der Gesetzgeber bei der Revision von KVG Art. 58 neben den Nationalen Qualitätsverträgen explizit Raum für andere Entwicklungen schafft. Das Bemühen der Health Professionals um Qualität ist zu fördern, nicht zu behindern. Es gilt zu vermeiden, dass sich Akteure mit Hinweis auf noch fehlende oder auf abstrakt formulierte Qualitätsverträge von Q-Aktivitäten zurückziehen. Dazu ist es auch zwingend, dass der Gesetzgeber die Umsetzung der Qualitätsaktivitäten einer von der Bundesverwaltung unabhängigen Organisation übergibt, welche Health Professionals und $\mathrm{Pa}$ tienten mit ihren Organen sowie Versicherer und Kantone in die strukturelle Verantwortung (z.B. in Form einer Stiftung, eines Vereins oder einer AG) und in die strategische Entscheidungsfindung miteinbezieht [18].

\section{Referenzen}

1 Harke JD (2007). Das Sanktionssystem des Codex Hammurapi, Würzburger rechtswisseschaftl. Schriften 70, Würzburg.

2 Kahn L, Corrigan J, Donaldson M. To err is human - building a safer health system, National Academic Press (2000), Washington DC.

3 OECD (2017a). Tackling Wasteful Spending on Health, OECD Publishing, Paris.

4 Trageser J, Vettori A, Iten R, Crivelli L (2012). Effizienz, Nutzung und Finanzierung des Gesundheitswesens, Akademien der Wissen schaften Schweiz, Bern.

5 Freidson E (2013). Professionalism: The Third Logic, John Wiley \& Sons, London.

6 Donabedian A (2003). An Introduction to Quality Assurance in Health Care, Oxford University Press, New York.

7 IOM Insitute of Medicine (1990). Medicare: a stratgey for quality assurance, Vol 1, National Academic Press, Washington DC.

8 PVK (2008). Die Rolle des Bundes bei der Qualitätssicherung nach KVG, Bericht der Palamentarischen Verwaltungskontrolle zuhanden der Geschäftsprüfungskommission des Ständerates, BBI 2008, 7797-7888.

9 BAG (2009). Qualitätsstrategie des Bundes im Schweizerischen Gesundheitswesen, EDI, Bern.

10 BAG (2014). Vorentwurf zum Bundesgesetz über das Zentrum für Qualität in der obligatorischen Krankenpflegeversicherung, EDI Bern

11 BAG (2015). Faktenblatt «HTA-Programm / HTA-Strategie und Zulassungsverfahren", EDI, Bern, August 2015

12 Nylenna M, Bjertnages O, Sperre Saunes I, Lindahl AK (2015). What is Good Quality of Health Care?, in: Professions and Professionalism, Vol 5, No 1, 1-16.

13 Güntert B, Offermanns G (2001). Qualitätsmanagementmodelle für das Gesundheitswesen, in: lögd (Hrsg.), Qualitätsmanagement im ÖGD, lögd, Band 9, Bielefeld, S. 13-3.

14 BVGE 2014/36. Grundsatzurteil vom Bundesverwaltungsgericht vom 11. September 2014.

15 SAMW (2011). Zertifizierung im medizinischen Kontext, Empfehlungen der Schweizerischen Akademie der Medizinischen Wissenschaften, Basel.

16 Oertle D. Die Hausärzte sterben aus: Na und? Schweiz Ärztezeitung. 2016;97(51-52):1804-1805.

17 Wolff E. Über Patientenschelte. Schweiz Ärztezeitung 2017;98(13):422.

18 OECD (2017b). Caring for Quality in Health - lessons learnt from 15 reviews of health care quality, OECD Publishing, Paris.

19 Sorgenfrei JH (2015). Qualitätsmanagement in der vertragsärztlichen Versorgung - Evaluation durch den Gemeinsamen Bundesausschuss, Diplomica Verlag, Hamburg. 